\title{
Miranda
}

Revue pluridisciplinaire du monde anglophone /

Multidisciplinary peer-reviewed journal on the English-

speaking world

$10 \mid 2014$

Images on the Move: Circulations and Transfers in film

\section{Phénomènes de circulations en festival : l'influence des nouvelles technologies}

\author{
Christel Taillibert
}

\section{OpenEdition}

Journals

Édition électronique

URL : http://journals.openedition.org/miranda/6330

DOI : 10.4000/miranda. 6330

ISSN : 2108-6559

Éditeur

Université Toulouse - Jean Jaurès

\section{Référence électronique}

Christel Taillibert, «Phénomènes de circulations en festival : l'influence des nouvelles technologies », Miranda [En ligne], 10 | 2014, mis en ligne le 23 février 2015, consulté le 16 février 2021. URL : http:// journals.openedition.org/miranda/6330; DOI : https://doi.org/10.4000/miranda.6330

Ce document a été généré automatiquement le 16 février 2021.

\section{cc) (i) () $\Theta$}

Miranda is licensed under a Creative Commons Attribution-NonCommercial-NoDerivatives 4.0 International License. 


\title{
Phénomènes de circulations en festival : l'influence des nouvelles technologies
}

\author{
Christel Taillibert
}

\section{Questions de circulation}

1 En termes de distribution cinématographique, tout du moins pour ce qui concerne l'ère désormais révolue de la pellicule analogique, la diffusion artistique a pendant longtemps été indissociable d'une circulation, matérielle, de copies de films dans les différents lieux de projection envisagés : salles de cinéma bien sûr, mais aussi salles des fêtes, ciné-clubs, plein-air, etc. La présence, indispensable à la projection, de cet objet symbolique, acheminé par des transporteurs spécialisés, et dont on savait aussi vanter la rareté, sacralisait le moment et le lieu choisis pour l'organisation de cette projection, et les unissait de fait dans le plaisir collectif de ce temps particulier. La circulation de la copie vers un point donné impliquait, de fait, la circulation du public vers ce lieu d'adoption.

2 Le développement progressif de la télévision, sa popularité grandissante et la chute de la fréquentation cinématographique à partir de la fin des années 1950 en France, vint bouleverser cette relation particulière entretenue à l'égard de l'objet-film, permettant dorénavant une réception individuelle, à domicile, d'une œuvre cinématographique qui circulait désormais par les voies immatérielles des canaux hertziens, câblés ou satellitaires. Les progrès technologiques qui ont marqué le dernier demi-siècle magnétoscopes, DVD, VOD, SVOD, streaming, etc. - ont certes accéléré la circulation des œuvres cinématographiques vers ses spectateurs potentiels, mais toujours selon des modalités de réception plus individualisées, personnalisées, loin du bouillonnement culturel et communautaire des séances collectives liées au grand écran.

3 Parmi les réponses qui furent historiquement apportées pour renouer avec des formes collectives de visionnement, tout en repensant ces questions de circulation - du public, 
des œuvres, du savoir - nous nous intéresserons ici à la question des festivals de cinéma. Ceux-ci furent, et ce n'est pas un hasard, l'objet d'un développement massif à partir des années quatre-vingt, et a fortiori quatre-vingt-dix, décennies marquées par la démultiplication de l'offre télévisuelle, désormais délivrée en France du monopole d'Etat, et des modalités d'accès alternatives aux images animées, avec l'apparition de technologies de plus en plus innovantes, autorisant à chaque fois plus de souplesse et de liberté dans le visionnement individuel d'images animées (Boudet-Dalbin). Ces manifestations tentaient, en effet, de réinvestir les valeurs mises à mal par l'individualisation galopante des pratiques culturelles - la convivialité, l'échange, le partage, la dimension communautaire de l'expérience prenant souvent le pas sur les questions de transmission artistique. Or, les très récentes évolutions dont témoigne le milieu des festivals instaurent un déplacement inattendu du concept de festival sur le terrain de la réception individuelle. On voit en effet fleurir depuis quelques années des festivals en ligne, proposant, par le biais d'un site internet, le téléchargement des œuvres cinématographiques programmées dans le cadre de l'événement. Si des horizons inespérés s'ouvrent sans conteste en faveur de la circulation des œuvres, le festivalier redevient dans cette nouvelle configuration un individu, seul devant sa machine.

4 L'objet de cet article est d'interroger cette évolution, d'évaluer la capacité de ce nouveau type de manifestation à demeurer un élément structurant face à une communauté devenue virtuelle donc fluctuante. Comment repenser la fonction de médiateur telle que l'ont historiquement pensée les festivals dans ce contexte de circulation accélérée et démultipliée des œuvres que propose leur avènement sur la Toile?

\section{Le festival, ou la circulation des œuvres et du public}

5 Dans un premier temps, revenons cependant à la forme traditionnelle autour de laquelle s'est construite la notion même de festivals de cinéma, afin de comprendre en quoi leur activité s'est singularisée dans le vaste territoire de l'exploitation et de la diffusion cinématographique. Ces manifestations apparaissent historiquement sous la forme d'événements nationaux, isolés, sur le modèle proposé en 1932 par la Mostra Internazionale d'arte cinematografica de Venise, et définitivement entériné par le Festival International $d u$ film de Cannes dans l'immédiat après-guerre. Ils mêlent alors des objectifs de promotion des industries cinématographiques nationales à la volonté de servir le prestige du pays qui les organisait ${ }^{1}$. Les bases sur lesquelles se construisent ces événements entremêlent ainsi des caractéristiques très différentes: un ancrage résolument mondain croisant un culte très populaire des vedettes, tous deux portés par une mise en scène grandiose, mais aussi une dimension commerciale incontournable, initialement portée par les compétitions, et qui s'accentuera avec la création des marchés du film (en 1959 à Cannes).

Parallèlement à ce premier modèle qui perdure jusqu'à nos jours, les festivals du film connaissent, timidement à partir des années soixante et soixante-dix, puis beaucoup plus massivement dans les années quatre-vingt et quatre-vingt-dix, un développement considérable sous la forme de manifestations plus modestes, davantage tournées en direction d'un public local et ciblant des typologies de films aux critères de plus en plus restreints. L'événementiel, en raison de sa promesse festive, de la notion d'exception qu'il induit, devient alors le moteur de l'émergence massive de ces nouveaux acteurs 
sur le terrain de la diffusion cinématographique - nouveau étant tout du moins le poids qu'ils représentent désormais sur le terrain de la diffusion dite non-commerciale. Transformer en événement la présence d'un film donné, dans un lieu particulier, devient le moteur d'une force par laquelle des spectateurs mus par des intérêts très variables se retrouvent à participer à une même manifestation.

7 En effet, du point de vue des publics, et toujours pour interroger cette question de circulation, ces événementiels travaillent à construire des liens communautaires sur la base de propositions et d'objectifs qui peuvent être très différents d'une manifestation à l'autre (Taillibert 45-87) :

- Certains mettent en avant la dimension cinéphilique de l'événement, qu'elle s'exprime de façon générale pour le cinéma dans son ensemble ou bien sur un créneau beaucoup plus exigu ; la richesse artistique de la programmation devient alors le point névralgique du pouvoir attractif de la manifestation ${ }^{2}$;

- D'autres choisissent de s'inscrire dans une thématique particulière, qui en fonction de sa teneur, s'avère susceptible de mobiliser certaines catégories de populations, soit au nom d'une passion - extra cinématographique - commune, soit au nom de revendications militantes, soit enfin en vertu d'une mission citoyenne, éducative, destinée à faire réfléchir le spectateur à propos du monde qui l'entoure à de la place que lui-même, en tant que citoyen, occupe dans ladite société ; la programmation est toujours ici proposée comme l'élément attractif en direction du public, mais c'est son contenu thématique plus que sa dimension artistique qui est mise en avant ;

- D'autres enfin choisissent de privilégier la dimension festive, la manifestation constituant avant tout la possibilité pour des individus issus d'une même communauté sociogéographique de tisser des liens entre eux, mais aussi avec les représentants du tissu socioculturel de leur cadre de vie. L'attractivité de la manifestation tient alors davantage à sa capacité à créer du lien social qu'à la programmation elle-même, que l'on peut davantage considérer comme un produit d'appel.

8 Ainsi, la circulation des publics, leur rassemblement dans un lieu investi d'une promesse événementielle, est au cœur du projet même des festivals tels qu'ils se sont historiquement construits. La présence d'invités prestigieux (réalisateurs, acteurs, professionnels divers, spécialistes des questions abordées par la thématique du festival...) compte bien entendu énormément dans la création de "l'événement », le public ressentant d'autant plus sa présence à la manifestation comme un privilège, et cette expérience partagée contribue à créer un sentiment fort d'appartenance à une communauté d'initiés entre les participants. Ceci n'ôte rien bien sûr à la richesse que proposent les festivals au regard de la circulation des images, qu'ils accueillent dans leur diversité, comme en témoigne la variété des productions audiovisuelles bénéficient aujourd'hui d'une circulation en festivals : fictions, séries, reportages, documentaires, clips, publicités... Ces initiatives conduisent, étonnamment, à rassembler du public autour du visionnement collectif d'œuvres audiovisuelles initialement conçues pour le petit écran, et donc pour un visionnement individuel.

Cette tendance, déjà significative pour ce qui concerne les productions audiovisuelles, est plus étonnante encore lorsque l'on se penche sur les autres types d'images animées auxquelles se consacrent aujourd'hui des festivals :

- toutes les productions amateur, réalisées grâce à caméscopes de toutes gammes ${ }^{3}$, mais aussi grâce à des appareils photos (festival Takavoir et Nikonfilmfestival, créés en 2010...), des téléphones portables (Mobile Film Festival créé en 2006, festival Pocket Films [2005-2010]...). 
- les productions pour le Web, à l'image par exemple du Web TV Festival de la Rochelle, créé en 2010 - festival présentant des productions destinées aux télévisions sur le Web-, ou encore le Marseille Web Fest, créé en 2011 à Marseille, présentant des Web-séries. Le va-et-vient entre l'écran de l'ordinateur et le besoin ressenti de montrer ces productions sur grand écran, devant un public en chair et en os, est très intéressant du point de vue des circulations, puisqu'il implique un retour paradoxal à un schéma traditionnel de visionnement pour des œuvres qui ont vu le jour grâce à la prolifération de réseaux diversifiés de circulation des images animées.

10 Ces différents développements s'inscrivent dans une démarche globale de résistance : résistance en termes de diversité culturelle, face à l'emprise des industries culturelles sur les marchés cinématographiques, mais aussi résistance face à la dislocation du lien social que favorise la dématérialisation totale de la filière cinématographique qui se préfigure (Mabillot). L'engouement dont furent l'objet les festivals de cinéma et audiovisuel au cours des dernières décennies semble ainsi démontrer l'existence d'un besoin de la part du public de renouer avec les formes collectives de visionnement des films, selon des modalités plus chaleureuses, plus enrichissantes aussi que celles que propose de son côté l'exploitation commerciale.

\section{Des festivals de cinéma en ligne}

11 L'apparition récente de nouveaux types de festivals, s'annonçant comme des festivals en ligne, remet totalement en cause toutes les propositions précédemment évoquées en termes de circulation. Ces manifestations proposent en effet à leurs usagers le visionnement de films en streaming ou par le biais du téléchargement, directement sur leur ordinateur personnel, leur téléphone, leur PDA, ou n'importe quel récepteur mobile. Cette pratique interroge la légitimité de l'appellation «festival», historiquement définie par l'idée du rassemblement d'un public dans un lieu précis, autour d'une action commune, sur un temps défini. Si la temporalité propre à l'événementiel de type "festival » perdure dans la forme "en ligne », s'il y a malgré tout une unité d'action dans l'événement proposé, la dématérialisation de l'événement, qui ne s'ancre plus dans un lieu géographique précis, ne permet plus d'associer à la manifestation l'idée de rencontres, dont on a vu à quel point elle était primordiale dans le succès de la formule. Afin de comprendre les tenants et les aboutissants de ces événementiels d'un nouveau type, nous évoquerons ici l'exemple d'une expérimentation française initiée par Unifrance, organisme chargé de la promotion à l'étranger du cinéma français. My French Film Festival n'est pas le premier festival de cinéma en ligne a avoir vu le jour : dès 2001, le Jameson Notodofilmfest était promu par le collectif espagnol « la fabricà », suivi par le Haydenfilms Online film festival aux Etats-Unis en 2004 , et d'autres encore à travers le monde jusqu'à aujourd'hui, mais sans toutefois que ce concept sorte véritablement de la marginalité.

\section{My French Film Festival : un succès grandissant}

12 C'est en 2011 qu'Unifrance organise son premier festival en ligne, intitulé My French Film Festival et qui, comme son nom l'indique, centre entièrement sa programmation autour du cinéma français, qu'il entend à travers cette initiative faire mieux connaître à l'étranger. Cet organisme travaille donc en étroite collaboration avec les 
professionnels français de l'industrie cinématographique, tentant à leurs côtés de défendre la cinématographie française à travers le monde, d'un point de vue à la fois culturel et économique, en particulier face à la très large domination des marchés américains dans le monde.

Composée de dix courts et dix longs métrages français en compétition, et de trois films hors compétition, la programmation de My French Film Festival est disponible par le biais du téléchargement sur le site du festival (http://www.myfrenchfilmfestival.com) - ou bien sur 20 plateformes partenaires à travers le monde. Dans certaines zones géographiques (l'Amérique latine, la Chine, la Pologne, la Russie et la Turquie), l'accès aux films est totalement gratuit pour les internautes, " grâce à la remontée aux ayants droits d'un forfait assuré par des partenaires privés » (Bilan 2012 3). Dans les autres régions, le téléchargement des films est payant, à des tarifs variables selon le pays dans lequel on se trouve ${ }^{4}$, différents packs étant parallèlement proposés («Pack long métrage », « Pack court métrage » ou « Pack Full Access »). Ces formules rappellent les abonnements traditionnellement proposés dans les festivals classiques, et constituent une première tentative pour renouer les deux concepts, autour de l'idée d'une fréquentation assidue de l'événement, elle-même liée au postulat cinéphilique ou affectif sur lequel se construit la relation entre une manifestation de ce type et ses (cyber)spectateurs. Lorsque les téléchargements n'étaient pas effectués directement sur la plateforme du festival (myfrenchfilmfestival.com), les principaux partenaires avec lesquels a collaboré Unifrance, en 2013, sont Youku/Tudu en Chine, Eurocinema aux Etats-Unis, Illico au Canada, Megogo.net en Russie, Orange en Pologne, Filmin en Espagne, Curzon en Grande-Bretagne, EuroVOD dans les autres régions d'Europe, et Itunes dans les autres régions du monde ${ }^{5}$.

Le développement de My French Film Festival a été fulgurant. Au cours de la première édition, 40.000 visionnages avaient été enregistrés de par le monde. En 2012, la seconde édition donna lieu à 1,3 million de visionnages, soit un résultat 30 fois supérieur à un an d'intervalle (Bilan 2012 3). La troisième édition ne réédita pas l'exploit, mais furent malgré tout comptabilisés 750.000 visionnages à travers le monde, ce qui reste un résultat très enviable puisque cette chute s'explique en réalité par la baisse notable des résultats enregistrés en Chine, inférieurs de près de 400.000 visionnages en 2013 par rapport à 2012, « du fait de la moindre visibilité accordée au festival sur la plateforme chinoise Youku en raison d'un changement d'équipe au sein du site " (Bilan 2013 3), alors même que les résultats enregistrés dans les autres parties du monde restent en progression.

Si l'on en revient aux objectifs premiers d'Unifrance - promouvoir le cinéma français à l'étranger - force est de reconnaître que le procédé est efficace, et qu'en termes de circulation - des œuvres - les résultats obtenus sont sans précédent. 188 pays ont été touchés lors de la troisième édition de la manifestation, la Chine restant le pays ayant le plus avidement participé à la manifestation, avec 375.000 de visionnages enregistrés (Bilan 2013 5) :

\begin{tabular}{|l|l|}
\hline Pays & Nombre de visionnages en 2013 \\
\hline Chine & 375.000 \\
\hline Pologne & 119.000 \\
\hline
\end{tabular}




\begin{tabular}{|l|l|}
\hline Russie & 61.000 \\
\hline Mexique & 40.994 \\
\hline Brésil & 38.830 \\
\hline Argentine & 17.500 \\
\hline Turquie & 17.000 \\
\hline Colombie & 15.000 \\
\hline Canada & 7.000 \\
\hline Etats-Unis & 6.500 \\
\hline Espagne & 4.500 \\
\hline
\end{tabular}

Du point de vue de la diffusion culturelle, motivation centrale de nombre de festivals de cinéma et audiovisuel, la version "en ligne » du concept semble donc prometteuse, puisque dans l'exemple qui nous occupe, elle a permis de faire faire connaître à des publics très divers les films à la programmation. Jean-Rémi Ducourtioux, chargé par Unifrance de l'organisation de cet événement, explique en ces termes la nature des motivations qui avaient prévalu au choix de l'intitulé «festival» pour qualifier cet événement impulsé par l'organisme pour lequel il travaille :

Il était important d'investir les nouveaux médias avec des formes déjà connues du public pour aller le chercher sur un terrain rassurant où il peut se projeter. L'objectif est le rajeunissement du public de cinéma français dans le monde, être disponible partout, pour tous, même pour ceux, toujours plus nombreux, qui n'ont plus accès à une salle diffusant du cinéma étranger (Ducourtioux).

17 Ainsi, le concept de festival en ligne semble répondre pour Unifrance à plusieurs exigences :

- recourir à une terminologie qui recouvre un concept connu et commun dans le monde entier, et dont la connotation partout positive : un festival, c'est avant tout un événementiel festif, bénéficiant d'une caution culturelle, et à laquelle participer apparaît comme un privilège. D'où l'adjectif « rassurant » utilisé par Jean-Rémi Ducourtioux: si la forme «en ligne» est nouvelle, la proposition s'inscrit par contre dans le cadre d'une expérience individuelle reconnue et appréciée ;

- pallier l'absence d'un réseau d'exploitation diffusant du cinéma français à travers le monde, et l'on retrouve là la proposition - inversée - qui sous-tend l'activité de nombre de festivals de cinéma en France, et qui cherchent, eux, à pallier la pauvreté de la diffusion de films étrangers sur le territoire national. Au sein de cette réflexion sur la circulation des films, on est donc bien en présence d'une innovation conceptuelle destinée à pallier l'absence de circulation des films français par les circuits classiques. Unifrance organisait déjà depuis de nombreuses années un certain nombre de festivals «classiques» (Le cinéma français aujourd'hui en Russie, le Festival du film français au Japon, le festival Varilux de Cinéma Français au Brésil, etc.), mais jamais ces manifestations n'avaient réussi à toucher des publics aussi nombreux et diversifiés ; 
- utiliser les nouvelles technologies pour capter un public jeune, consommant quotidiennement des images animées sur les nouveaux médias, et qui se montrera donc sensible à une telle offre. La question de la formation, de l'éducation des nouvelles générations, elle aussi centrale dans l'activité des festivals «classiques », est donc ici aussi au cœur de ce projet, mettant la question de la circulation des savoirs, des cultures, au cœur de l'événement.

\section{La réappropriation du modèle festivalier}

Au-delà de ces premiers éléments fondamentaux en termes d'objectifs, les modalités de développement de la proposition en ligne sont totalement conçues pour se fondre dans le moule historique "festival», pour tisser des liens entre des formes que tout semble opposer de par la virtualisation du concept. Ces choix sont aussi clairement destinés à différencier l'offre proposée par un «festival en ligne» de ce que proposent de leur côté les multiples sites de téléchargement, cinémathèque en ligne, etc. :

- tout d'abord, en termes de temporalité, la manifestation s'inscrit dans une période limitée, un moment privilégié en dehors duquel l'accès au film n'est plus possible ; l'édition 2013 s'est ainsi déroulée du 17 janvier au 17 février, soit sur une période d'un mois ;

- la ligne artistique du festival est clairement définie, puisqu'il s'agit d'éclairer la jeune création française dans le domaine cinématographique : les films proposés ne sont donc pas les films qui ont le mieux fonctionné au box-office, ou qui bénéficient des financements les plus imposants, mais bien des films sélectionnés en vertu de leur originalité, de leurs qualités artistiques, donc sur la base de critères que l'on peut qualifier de cinéphiliques ;

- les films sont proposés en versions originales sous-titrées (les sous-titres étant disponibles en 12 langues $^{6}$ ), ce qui satisfait les exigences de respect artistique de l'œuvre telles qu'elles sont pratiquées par la très grande majorité des festivals ;

- le site propose, en accès libre et pour chaque film proposé, des fiches techniques, mais aussi des interviews exclusives des réalisateurs et acteurs ayant participé au projet. Cette disposition répond à une caractéristique fondamentale des festivals classiques, lesquels affichent aussi leur singularité sur le terrain de la diffusion cinématographique par leur capacité à animer les projections, à offrir des prolongements à la réflexion du spectateur par le biais de documents d'accompagnement, de rencontres de professionnels, etc...;

- une compétition, caractérisant le fonctionnement de nombre de festivals, et tout particulièrement des festivals de cinéma les plus prestigieux, est organisée autour des 20 films en compétition. Conformément au fonctionnement classique des festivals de cinéma, trois jurys différents donnent lieu à quatre prix dans chacune des deux catégories. Le prix de la Presse Internationale est délivré par dix journalistes influents en provenance du monde entier, sa présence constitue une caution intellectuelle à la manifestation, selon un schéma classique dans ce milieu. Le prix des Cinéastes étrangers est délivré par quatre cinéastes, et permet ainsi de donner une voix à la profession. Le Prix des réseaux sociaux est quant à lui délivré par des personnalités influentes sur les réseaux sociaux dans le monde entier, au nombre de 100 en 2013, qui vont jouer un rôle de relais dans les différents pays concernés, pour inciter les internautes à découvrir cette initiative. C'est une façon bien entendu de mettre à l'honneur les évolutions induites par Internet en termes de communication, de pratiques cinéphiliques, d'alternatives participatives. Enfin, un quatrième prix, dit Prix du public, permet cette fois d'impliquer les cyberspectateurs en les invitant à voter pour leur film préféré, ce qui permet, malgré cette géographie éclatée, de reconstruite les bases d'une communauté, quand bien même virtuelle, d'intérêt. 
C'est bien évidemment cette dimension qui s'avère la plus délicate dans l'utilisation du vocable «festival» dans le cadre de cette évolution en ligne: l'intérêt d'une telle manifestation, dans sa version classique, repose beaucoup sur l'interaction qu'elle autorise entre les participants, de façon formelle au cours de débats organisés à l'issue des projections, au cours de buffets ou repas en commun, etc., mais aussi de façon informelle dans les couloirs, halls d'accueil, buvettes, etc. La dématérialisation de l'événement, par définition, en abrogeant toute circulation des publics, a rendu nécessaire le recours à d'autres moyens pour permettre aux spectateurs d'interagir malgré tout. La solution adoptée par My French Film Festival consiste à animer sur son site un forum de discussion sur lequel chaque participant peut formuler « des avis et commentaires sur les films» («Conditions générales applicables à l'accès au site web Myfrenchfilmfestival.com et à l'utilisation de ses services»). L'objectif de la part des organisateurs est de favoriser l'émergence d'une réflexion collaborative entre les participants, leur permettant de "gérer des activités intellectuelles collectives " pour reprendre l'expression de Michel Marcoccia (3), qui envisage la possibilité pour de tels forums de proposer de nouvelles « formes de cognition collective» (Marcoccia 3), dans le cas qui nous occupe autour de la question cinématographique. De prime abord, si un tel forum peut générer une ébauche de communication entre les participants, la question du lien social est plus délicate, pouvant laisser craindre, comme l'écrivait Philippe Breton « un double risque - terrible - de solitude des personnes et de collectivisation de leur pensée » (Breton 108).

Il est évidemment très difficile de jauger, de comprendre les modalités de l'usage réel par les internautes de cette proposition, au-delà du projet tel qu'il a été dessiné par les concepteurs de cette manifestation. C'est pourquoi, afin d'évaluer le rapport entretenu par le public des premières éditions de My French Film Festival avec ce nouveau concept d'événementiel en ligne, une enquête a été menée auprès de ce dernier par le biais d'un questionnaire en ligne, du 20 mars au 17 avril 2013, donc à l'issue de la troisième édition du festival. Le lien vers le questionnaire, accueilli par la plateforme de sondage Interceptum, a été transmis par email aux usagers des trois premières éditions de la manifestation. 2539 questionnaires ont été intégralement remplis ${ }^{7}$, depuis 60 pays différents à travers le monde ${ }^{8}$, et ont donc constitué le corpus d'étude sur la base duquel sont proposées les pistes de réflexion suivantes.

\section{Le public de My French Film Festival et le concept de festival en ligne}

21 Plusieurs résultats dans cette enquête peuvent ouvrir des pistes de réflexion relatives aux la façon dont les internautes ont accueilli, au niveau mondial, cette initiative. Notons tout d'abord la globale jeunesse du public de My French Film Festival : près de la moitié (48,3\%) des festivaliers qui ont répondu à l'enquête ont moins de 35 ans (fig. 1), résultat qui semble rencontrer l'objectif de rajeunissement du public du cinéma français énoncé par Unifrance à l'égard de cette manifestation. 
Fig. 1

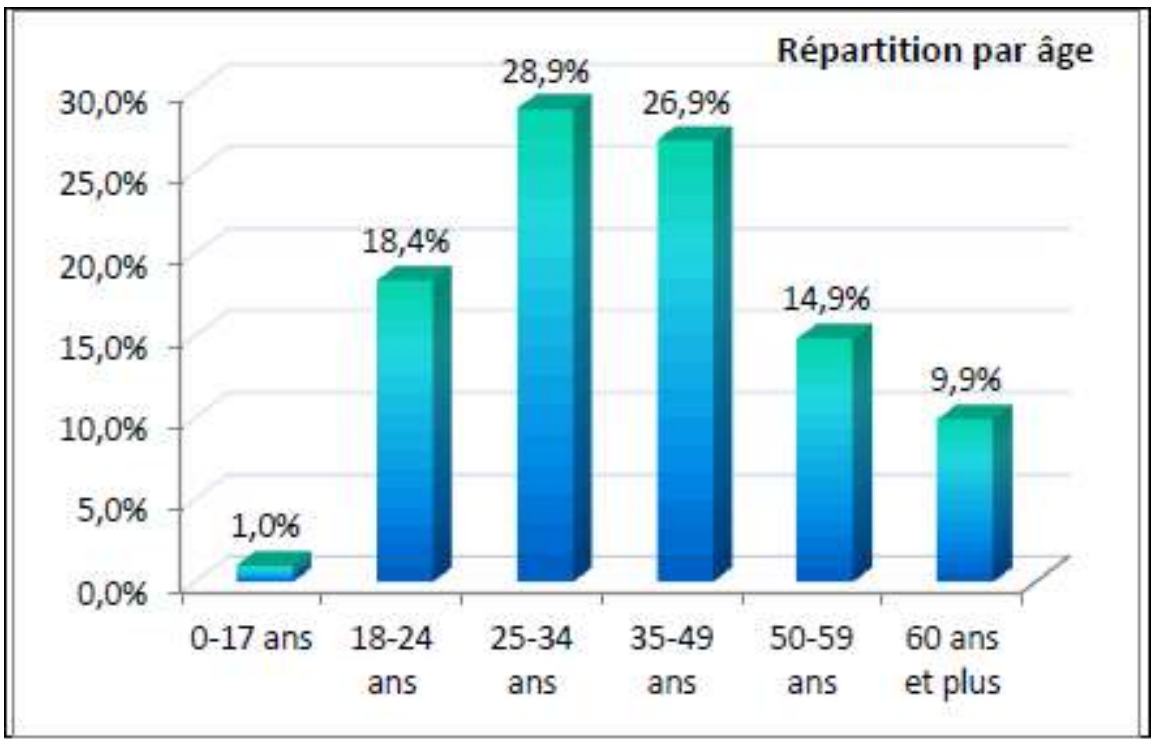

22 Au-delà de cette première et heureuse concordance, ce phénomène est surtout intéressant au regard des caractéristiques globales du public du cinéma français à travers le monde, particulièrement vieillissant. Si, déjà, ce phénomène est observable sur le sol français (CNC 66), il est encore plus marqué à l'étranger (Vincendeau 2009). En termes de circulation des œuvres, le concept de festival en ligne pourrait donc constituer un agent positif en faveur de la diversité culturelle, puisqu'il semble susceptible d'ouvrir à des œuvres spécifiques, peu diffusées, des publics nouveaux, a priori peu investis sur ces créneaux en termes de consommation culturelle.

Dans un même mouvement, à peine plus d'un tiers des participants à My French Film Festival ayant répondu au questionnaire fréquente des festivals de cinéma sous leur forme classique (fig. 2). Ceux-là, déjà sensibilisés à la forme festivalière à envisagent la création de festivals en ligne comme une pratique cumulative, susceptible d'enrichir leurs expériences et leurs découvertes cinématographiques. Pour les nombreux autres $(65,7 \%)$, cette expérience constitue un moyen de découvrir le concept de festival grâce à cette forme en ligne. De nouveau, cette capacité à atteindre de nouveaux publics ouvre de nouveaux horizons aux politiques de démocratisation culturelle telles qu'elles se sont jusqu'alors appliquées au cinéma. 
Fig.2

Fréquentez-vous des festivals de cinéma " classiques " ?

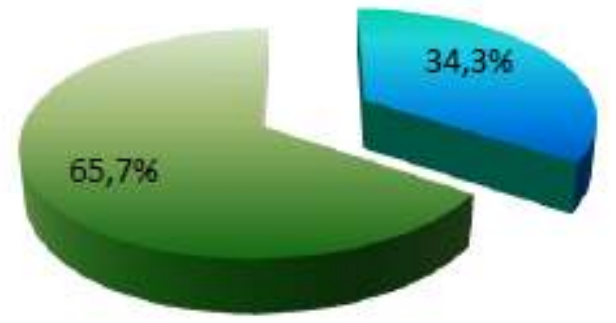

Comment expliquer la capacité de cet événement à toucher des publics nouveaux, non initiés à la forme festivalière ? La question de la souplesse que confère au concept de festival le recours aux nouvelles technologies est le facteur essentiel qui explique cette adhésion, puisque $71,1 \%$ des participants à l'enquête mettent ce point en avant pour qualifier leur perception de la notion de «festival en ligne » (fig. 3). Ainsi, c'est bien la liberté dans le rapport à la programmation que permet l'outil Internet (visionnement à toute heure, de n'importe quel endroit, possibilité de fractionner le visionnement, etc.) qui est au cœur du renouveau du concept même de festival, tout de moins dans la perception qu'en ont les usagers.

Cette première affirmation pourrait laisser penser que les utilisateurs de My French Film Festival considèrent cette manifestation comme une plateforme de téléchargement, au sein de laquelle ils puissent les films qu'ils souhaitent visionner, comme ils le feraient sur n'importe quelle interface offrant en ligne des documents cinématographiques. Cependant, 43,9 \% des internautes interrogés précisent aussi que la dimension événementielle - intimement liée au concept même de "festival» - joue un rôle important d'émulation dans leur démarche (fig. 3). A une époque où se multiplient les lieux de ressources sur la toile, qui tendent à mettre à la disposition du grand public un nombre de produits culturels croissant dans tous les domaines, ce résultat pointe l'importance de guider le public dans ses démarches culturelles, de l'inciter à aller à la rencontre de tel ou tel objet culturel plutôt qu'un autre, donc d'organiser des circulations entre des œuvres sélectionnées. En cela, les festivals en ligne semblent donc susceptibles de jouer, bien que sur un autre plan, un rôle de médiation similaire à celui que poursuivent les festivals classiques, dans la mesure où ils semblent susceptibles de donner du sens à la manne inépuisable mais nébuleuse que constitue Internet dans le champ culturel.

Dans des proportions quasi identiques, $41,4 \%$ des internautes interrogés déclarent ressentir une émulation intéressante du fait que cette manifestation leur confère un sentiment d'appartenance à une communauté internationale (fig. 3). A l'opposé, seuls $4,6 \%$ des internautes affirment qu'un festival en ligne n'est pas véritablement un festival, justement parce que manque cette dimension communautaire (fig. 3). Ces résultats relativisent donc la dimension initialement solitaire du visionnement d'un film sur Internet, qui en cela s'oppose directement au caractère collectif inhérent à la 
projection en salle ou tout autre lieu public, tels que la pratiquent les festivals classiques. Ils démontrent que les nouveaux «cyber-festivaliers " développent une conscience accrue du caractère communautaire de l'expérience qu'ils vivent dans le cadre d'un événement particulier. Cette expérience que l'on sait partagée acquiert donc une saveur particulière, et un goût pour l'échange.

Fig.3

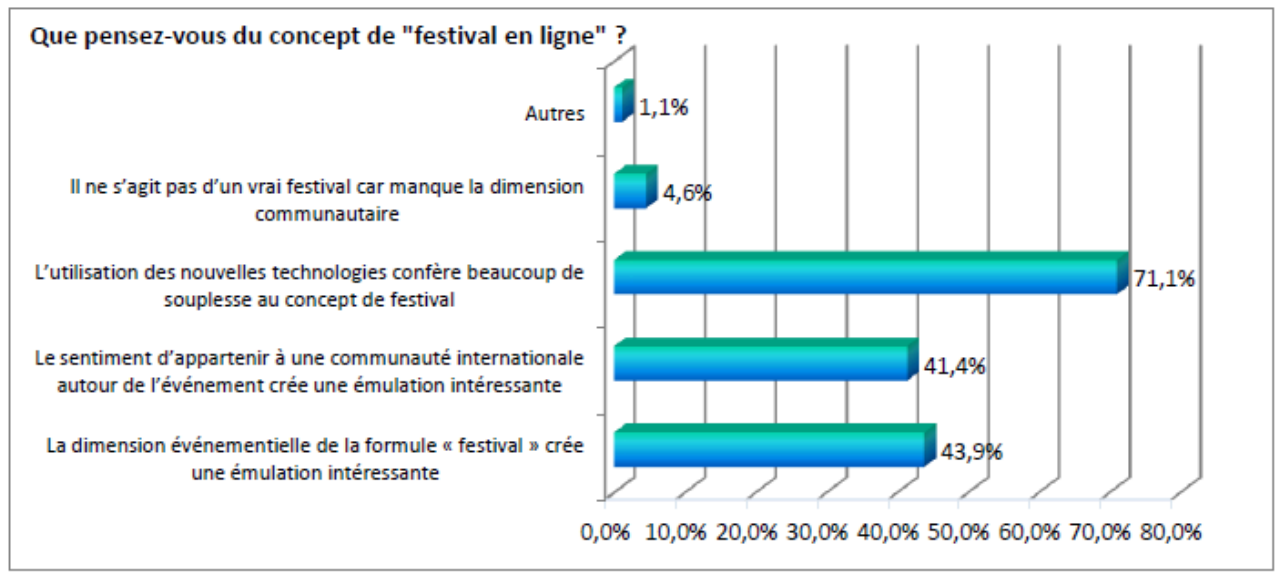

Nota Bene: le total des pourcentages est supérieur à 100 en raison des réponses multiples possibles

Comme évoqué précédemment, l'outil mis à la disposition des internautes pour donner corps à ce sentiment communautaire réside essentiellement dans l'animation d'un forum, espace d'échange et d'expression pour les festivaliers. Seule une minorité d'internautes interrogés (12\%) déclare avoir participé au forum, en postant des commentaires sur les films visionnés (fig. 4). Par contre, les internautes qui lisent les commentaires postés sur le forum représentent un peu plus de la moitié du corpus (fig. 5). De nombreux festivaliers s'intéressent donc aux avis de la minorité de participants que nous identifions précédemment, sans ressentir pour autant le besoin de s'exprimer eux aussi. Ces données se rapprochent finalement de ce qui peut se produire au sein d'un festival dit classique : tous les spectateurs ne restent pas pour assister aux débats qui peuvent être organisés à l'issue de la projection, et parmi ceux qui restent, une minorité va s'exprimer au cours dudit débat. Dans la version « en ligne » du festival, on retrouve par le biais de l'usage qui est fait du forum un espace interactif qui est investi plus ou moins activement en fonction du caractère et des attentes des usagers. Ces résultats sont en outre à appréhender au regard des résultats de fréquentation globale de la manifestation : sur les 750.000 participants à l'édition 2013, on peut estimer sur la base des résultats à cette enquête que 90.000 personnes ont choisi de s'exprimer à propos de leurs ressentis à propos de la programmation, résultat qui témoigne en faveur de la capacité d'Internet à galvaniser l'expression personnelle - témoignages, émotions ou expressions empruntant davantage à la culture savante en matière de critique cinématographiques - dans un élan propre l'émergence d'un désir de découverte, d'échange, de confrontation. 
Fig.4

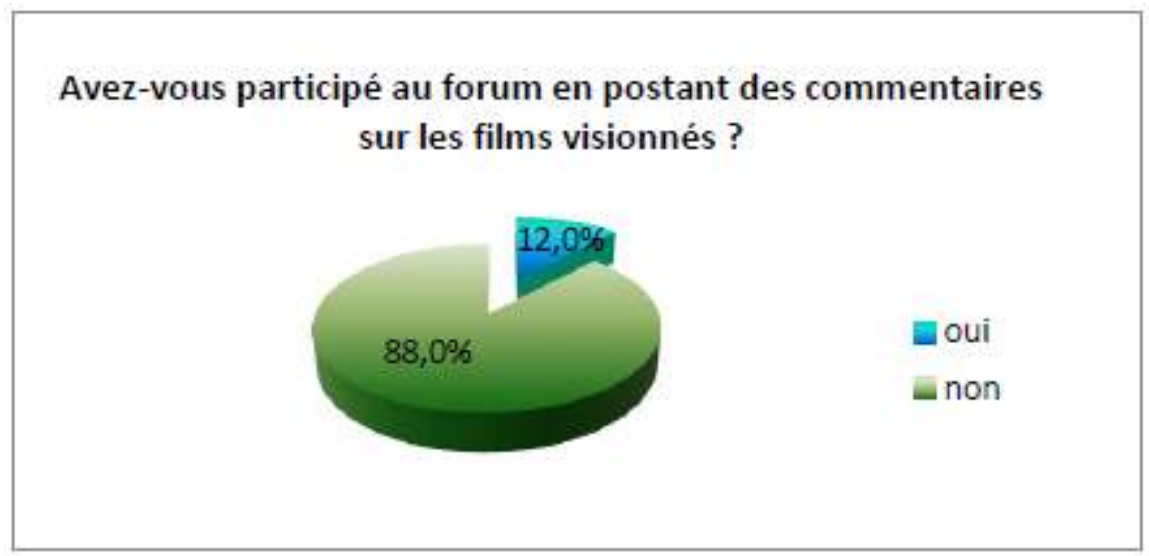

Fig.5

Avez-vous lu les commentaires postés sur le forum ?
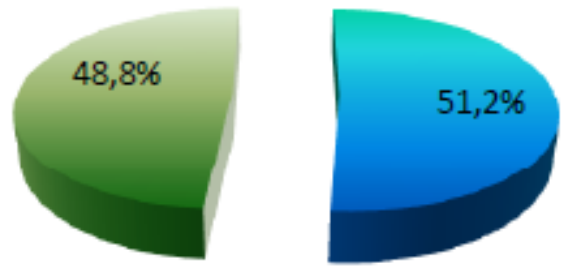

E oui

non

Le forum susmentionné n'est pas le seul espace au sein duquel les internautes interagissent, les réseaux sociaux constituant en particulier un prolongement évident de cette activité. Ainsi, bien que créée en janvier 2013 seulement, la page Facebook du festival (https://www.facebook.com/MyFrenchFilmFestival) a été visitée par $44,2 \%$ des participants au moment de l'enquête, soit quelques mois plus tard seulement (fig. 6). 72,5 \% des internautes concernés l'ont alors d'ores-et-déjà intégrée à leurs contacts. 


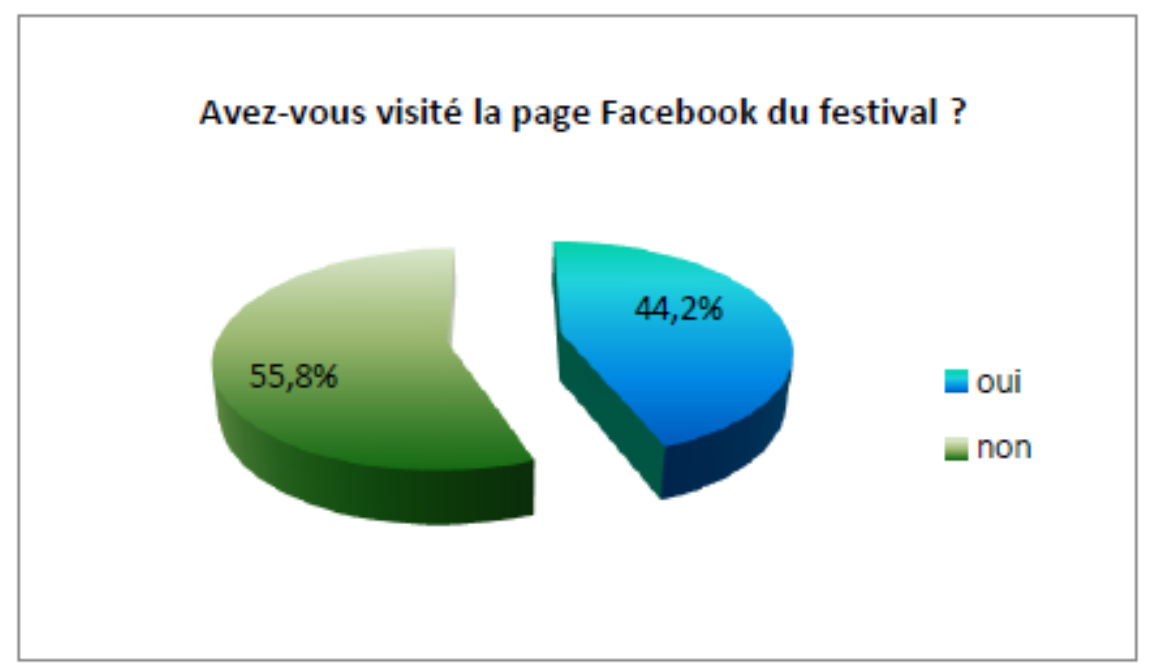

Fig.7

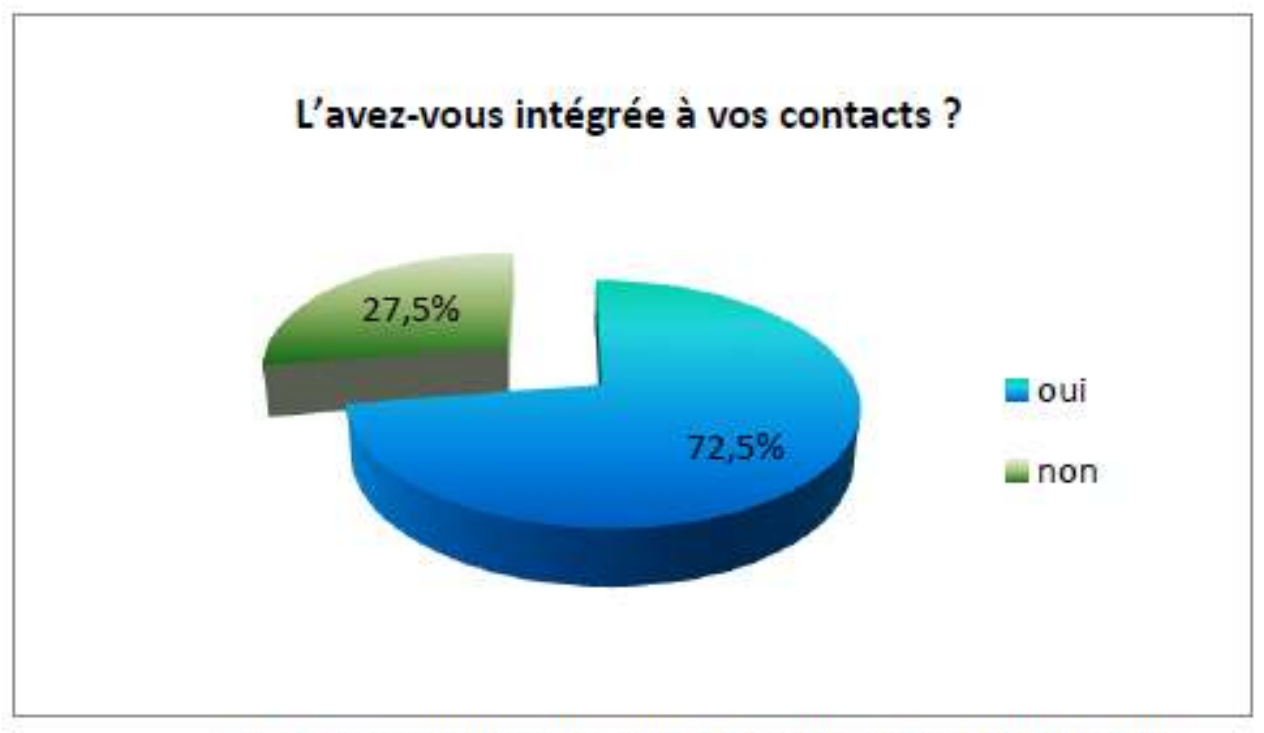

Pourcentages calculés sur la base des réponses des 1122 internautes ayant visité la page Facebook de My French Film Festival

Ces pratiques sont intéressantes dans la mesure où elles permettent véritablement de distinguer l'usage fait par ces cyberfestivaliers de ce nouveau type de manifestation en ligne de ce qu'offre une simple plateforme de téléchargement ou de consultation en streaming dans le domaine du cinéma: il semblerait malgré tout que la dimension communautaire constitutive du concept de festival trouve les moyens - sous des formes alternatives - de se manifester dans cette version en ligne. Pour approfondir cette question, il est intéressant d'observer que 6,9\% des participants à l'enquête déclarent avoir tissé des liens avec d'autres internautes par le biais du festival (fig. 8). L'expression «tisser des liens» reste ici bien évidemment assez vague, mais suppose d'un échange personnel - par email dans la très grande majorité des cas - ait été occasionné par la manifestation. Ce phénomème participe de la création de communautés virtuelles, fondée sur un intérêt commun pour le cinéma, et plus précisément pour le cinéma français, les groupes se constituant alors atour du 
«partage de valeurs, d'idéaux, d'objectifs et de représentations communes " (Bonfils, 117) autour de cette cinématographie particulière. Le rôle des réseaux sociaux est encore une fois fondamental dans la configuration de ces nouveaux réseaux de sociabilisation - quand bien même virtualisée - puisque 59,1\% des internautes qui ont déclaré avec tissé des liens avec d'autres participants affirment les avoir intégrés à leurs propres réseaux sociaux (fig. 9).

Fig. 8

\section{Avez-vous tissé des liens avec d'autres internautes participant au festival ?}

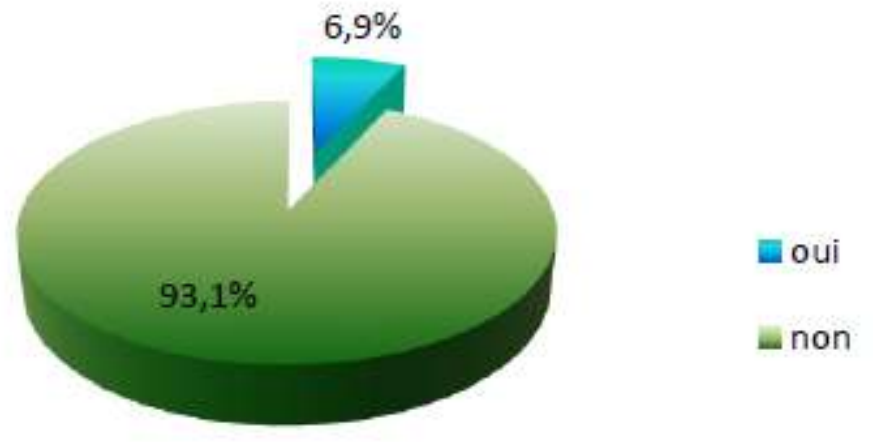

Fig. 9

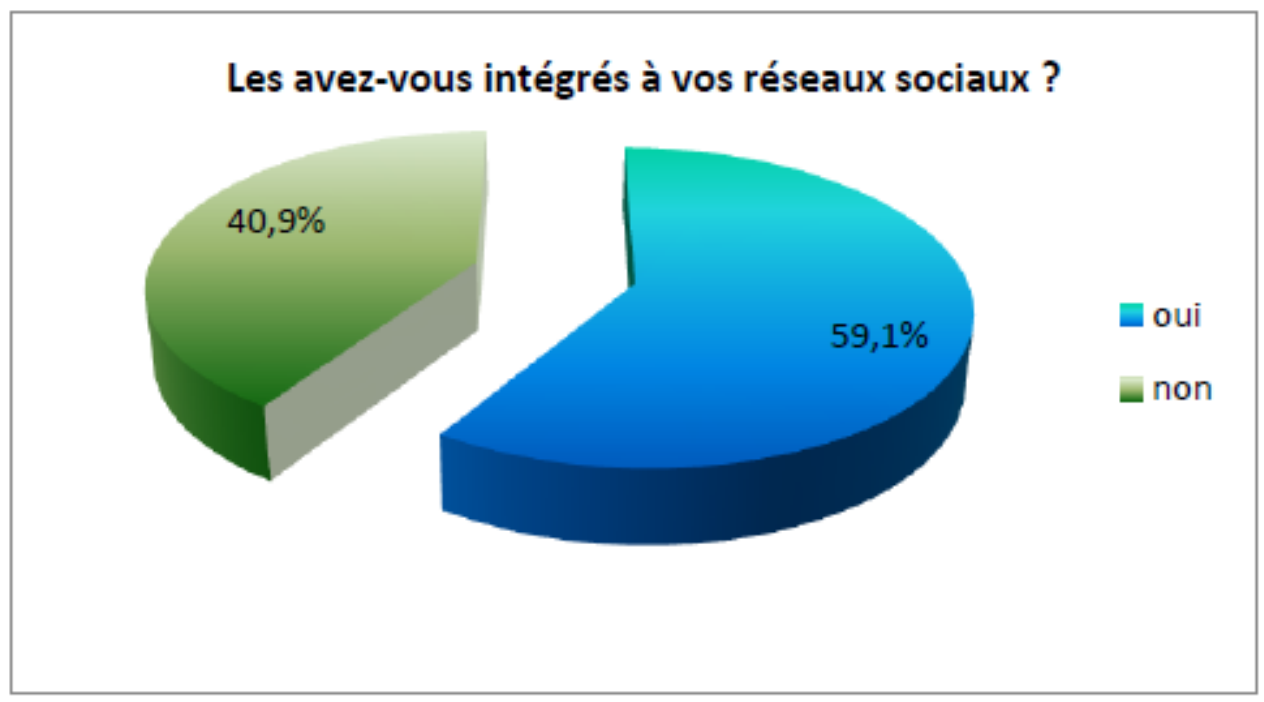

\section{Pourcentages établis sur la base des réponses des 176 internautes concernés par la création de liens par le biais du festival}

31 Ainsi, la notion de festivals en ligne correspondrait bien dans l'esprit des internautes à un concept bien particulier d'espace de rencontre avec des films, entièrement construit sur la base d'une expérience - vécue ou imaginée - des festivals de cinéma de type classique. Au-delà de la découverte artistique, la question communautaire semble en effet occuper une place conséquente dans la démarche du cyberfestivalier. D’ailleurs, 
les participants à My French Film Festival semblent tout particulièrement désireux de prolonger cette expérience participative, puisque $71,1 \%$ d'entre eux déclarent souhaiter que la communauté constituée par le public de cette manifestation puisse vivre tout au long de l'année, en dehors du festival (fig. 10). Ainsi, malgré l'attachement évident des internautes qui se sont exprimés dans cette enquête aux spécificités d'usage conférées par les nouvelles technologies, l'expérience collective que constitue cet événement est bien au cœur de sa réussite, et rapproche donc bien davantage le concept de festival en ligne d'une plateforme collaborative, centrée sur le cinéma français, que d'une plateforme de téléchargement aux perspectives plus consuméristes.

Fig. 10

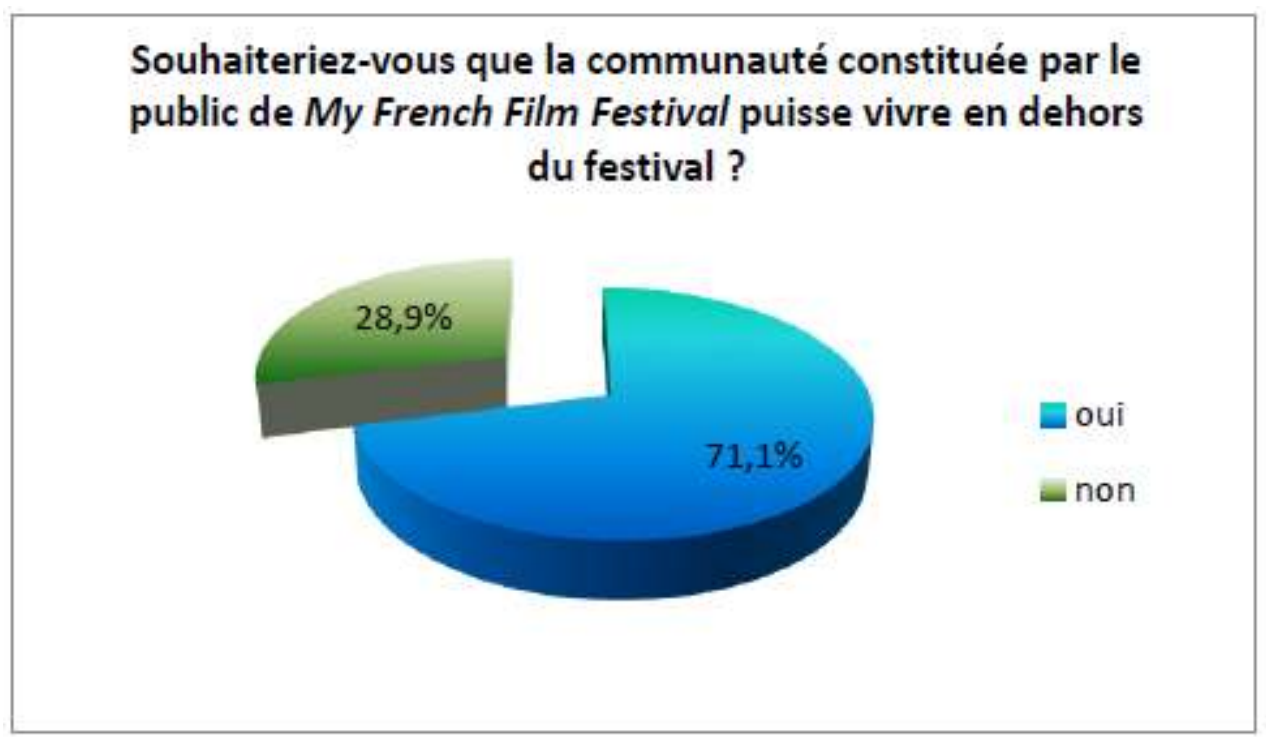

32 Ces constatations nous intéressent dans la mesure où la question de circulation que nous interrogions en préambule de cette étude prend ici un sens nouveau, dans la mesure où ces communautés virtuelles engendrées par l'activité de ce festival en ligne sont appelées à fonctionner comme des espaces de circulation de micro-cultures cinématographiques à travers le monde. L'éloignement géographique supposé des internautes est peu propice à la mise en place de relations profondes (Hérault, Molinier 19), mais les liens créés peuvent générer une émulation apte à dynamiser en profondeur la circulation des informations, des savoirs, des films, et donc à donner corps à la notion de diversité culturelle dans des parties du monde où elle est particulièrement mise à mal, en particulier dans le secteur cinématographique.

\section{Conclusion}

La déclinaison du concept de festival dans un mode virtuel, dématérialisé, oblige à repenser totalement les caractéristiques de ce type d'événementiels, leurs rôles en termes de circulation. Certes, l'exemple de My French Film Festival témoigne de l'incroyable potentiel de cet événement - pourtant encore très jeune - en faveur de la circulation des œuvres cinématographiques. Au cours de leur histoire, les festivals ont souvent réfléchi aux moyens dont ils disposaient pour élargir leur champ d'action, conscients du nombre réduit de spectateurs auxquels s'adresse un travail de diffusion 
qui a souvent occasionné un lourd travail de sélection. Nombreux sont ceux qui, en conséquence, ont joué la carte de la délocalisation de toute ou partie de leur activité : à l'intérieur même de leur ville d'influence, dans les communes limitrophes, au sein du département ${ }^{9}$, de la région, voire au niveau national ou international pour les plus importants d'entre eux ${ }^{10}$, mais quelle que soit la solution choisie, la démultiplication du public potentiel reste limitée par le nombre de structures mobilisées et leur capacité d'accueil respective. Aussi, la transposition du concept de festival sur la Toile apparaîtelle dans cette optique comme un moyen formidable de contourner cet obstacle inhérent au présentiel, en offrant à un nombre illimité de spectateurs la possibilité d'accéder, au même moment et dans une dynamique commune, à des œuvres sélectionnées.

Cette idée de (quasi) simultanéité propre à la notion d'événementiel est importante dans la mesure où elle conditionne la possibilité pour les internautes concernés de développer un sentiment d'appartenance commune à groupe restreint, groupe qui pourra éventuellement d'interagir autour de cette expérience collective, évoluer en une communauté virtuelle choisie et réfléchie, apte à faire vivre tout au long de l'année une passion commune, voire sur le moyen terme de construire ensemble une culture collective.

Loin de s'opposer aux festivals "classiques", leur déclinaison en ligne ouvre des horizons nouveaux susceptibles de prolonger sur Internet une mission éducative, une action de médiation qui a toujours structuré la raison d'être de ces manifestations. La démultiplication de l'offre en termes d'images animées sur Internet, disponibles le plus souvent gratuitement, a rendu impérieusement nécessaire de poser des jalons, d'accompagner la consommation d'œuvres en lui donnant un cadre culturel, mais aussi, malgré tout, un environnement humain - quand bien même virtualisé. Le concept de festival, parmi d'autres, peut constituer un moyen de donner forme à ce travail de médiation appliquée à la diffusion artistique, contribuant par la même à favoriser l'élaboration de nouvelles communautés de spectateurs, communiant autour de valeurs cinéphiles renouvelées, susceptibles de rallier de nouveaux publics aux besoins de l'art cinématographique. Bien sûr, rien ne remplacera le cadre festif, le bouillonnement culturel et l'incroyable convivialité à l'œuvre dans les festivals traditionnels, qui resteront des acteurs de proximité incontournables de la vie culturelle locale. Mais l'on peut parier sur le développement parallèle de manifestations en ligne, dont la fréquentation sera additionnelle et non substitutive aux festivals classiques, et qui offriront un terrain de jeux inégalé aux acteurs de la diffusion culturelle: les distributeurs et vendeurs à l'international y verront un moyen extraordinaire de diffuser lucrativement leurs produits dans des régions du monde où la cinématographie française ne parvient pas à s'imposer en salles, et les acteurs de la diffusion noncommerciale y trouveront un outil supplémentaire pour créer des liens entre des nouveaux publics et des œuvres cinématographiques et audiovisuelles atypiques et méritantes. 


\section{BIBLIOGRAPHIE}

Baeza, Cecilia et al. «L'invention de la contestation transnationale par les forums et sommets : la naissance d'un espace public mondial ?». Raisons politiques: 19:3 (2005): 25-43.

Bonfils Philippe. «Communication non verbale et avatars au sein d'une communauté virtuelle ». Quaderni: 73:3 (2010): 115-128.

Boudet-Dalbin, Sophie. « La distribution des films par internet : enjeux socioculturels, économiques et géopolitiques ». Université Panthéon-Assas, 2011.

Breton, Philippe. Le culte de l'Internet. Paris : La Découverte, 2000.

Centre national de la cinématographie et de l'image animée. Bilan 2012. Paris : Les dossiers du CNC, n³26, mai 2013.

Cholet, Mona, Rivière, Philippe (dir.). Internet, révolution culturelle. Paris : Le Monde diplomatique, Manière de Voir, février-mars 2010, nº109.

De Valck, Marijke. Film Festivals: From European Geopolitics to Global Cinephilia. Amsterdam: Amsterdam Univ. Press, 2007.

Donnat, Olivier. « Pratiques culturelles et usages d'Internet ». Culture/Etudes 11 (2007).

Hérault, Adeline, \& Molinier, Pierre. « Les caractéristiques de la communication sociale via Internet ». Empan: 76:4 (2009): 13-21.

Ethis, Emmanuel. Aux marches du Palais. Le Festival de Cannes sous le regard des sciences sociales. Paris : La Documentation française, 2001.

Ethis, Emmanuel. Sociologie du cinéma et de ses publics. Paris : Armand Colin, 2005.

Galland, Olivier. «Individualisation des mœurs et choix culturels », in Donnat, Olivier, Tolila, Paul. Le(s) public(s) de la culture. Paris : Presses de Sciences Po « Académique », 2003. 87-100.

Lescure, Pierre. Contribution aux politiques culturelles à l'ère numérique. Paris : Ministère de la culture et de la communication, mai 2013.

Latil, Loredana. Le Festival de Cannes sur la scène internationale. Paris : Nouveau Monde Editions, 2005.

Mabillot, David. La « menace fantôme » ou la numérisation du cinéma. Observatoire des mutations des industries culturelles, Série "Structures et stratégies financières et industrielles ", 2006. Consulté le 10 mai 2013 <http://www.observatoire-omic.org/fr/art/140/la-menacefantome-ou-la-numerisation-du-cinema.html>

Marcoccia, Michel. «L'animation d'un espace numérique de discussion : l'exemple des forums usenet ». Document numérique: 5:3 (2001): 11-26.

Proulx, Serge, Goldenberg, Anne. «Internet et la culture de la gratuité ». Revue du Mauss. 35 (2010).

Quillard, Marion. « Le réel en vingt clics ». XXI (2013): 28. Consulté le 5 septembre 2013. <http:// www.revue21.fr/Le-reel-en-vingt-clics>

Régnier, Isabelle. « Deux nouveaux festivals sur Internet » . Blog du Monde.fr (17 janvier 2011). Consulté le 15 mai 2013. <http://cinema.blog.lemonde.fr/2011/01/17/deux-nouveaux-festivalssur-internet/> 
Taillibert, Christel. Tribulations festivalières - Les festivals de cinéma et audiovisuel en France. Paris :

L'Harmattan, 2009.

Vincendeau, Ginette. «Le rayonnement du cinéma français vu par un Français ». lemensuel.net (13 mai 2009). Consulté le 15 mai 2013. <http://vupar.org/2009/12/19/le-cinema-francais-vu-par-lesbritanniques/>

\section{Autres sources citées}

Ducourtioux, Jean-Rémi. Interview réalisée par l'auteur, par email. 25 septembre 2012.

My French Film Festival. Bilan. Paris : Unifrance, 2012.

My French Film Festival. Bilan. Paris : Unifrance, 2013.

\section{NOTES}

1. On pourra utilement se référer à ce propos à l'ouvrage de Loredana Latil, offrant une passionnante analyse de l'histoire du Festival International $d u$ film de Cannes, particulièrement symptomatique de ces directions empruntées par les grands festivals internationaux jusqu'aux années soixante. L'ouvrage de Marijke De Valck propose par ailleurs une vision plus globale de ces évolutions historiques.

2. Citons pour exemple, en France, le Festival International du film de la Rochelle, Premiers plans à Angers, Itinérances à Alès, etc., parmi les festivals très généralistes; mais on retrouve cette tendance auprès de festivals spécialisés dans le court métrage (Festival International du court métrage à Clermont-Ferrand, Festival Tous courts à Aix-en-Provence, etc.), ou sur d'autres créneaux beaucoup plus atypiques (L'étrange festival, sur le créneau du film fantastique et d'horreur, en offre un exemple).

3. Cette tendance des festivals à rendre compte de la créativité des amateurs existait déjà avec le matériel analogique de format réduit, et a donc accompagné l'évolution du matériel de prise de vues.

4. Ainsi en France, il faut payer 3,99 € pour visionner un long métrage, et 0,99€ pour un court métrage. A l'étranger, ce tarif s'élève à $1,99 €$ pour un long métrage et $0,99 €$ pour un court métrage (source: «Conditions générales applicables à l'accès au site web myfrenchfilmfestival.com et à l'utilisation de ses services », article 3, http:// www.myfrenchfilmfestival.com/fr/cus

5. Pour un aperçu plus complet des multiples partenaires avec lesquels a travaillé Unifrance, on pourra se référer aux pages 16 et 17 du bilan 2013 de la manifestation.

6. Les films sont accessibles en allemand, anglais, arabe, chinois, espagnol, français, italien, japonais, polonais, portugais, russe et turc.

7. En l'absence de statistiques précises de fréquentation globale de la manifestation, il est difficile de proposer des chiffres précis quant à la représentativité de ce corpus. Quelques pistes peuvent cependant être proposées, quoique faussées par le fait qu'elles prennent en considération des résultats eux-mêmes tirés de l'enquête dont il est question : en 2013, le festival a bénéficié de 375.000 visionnages. L'enquête menée a montré qu'en moyenne, chaque cyberspectateur avait visionné 8,5 films (courts et longs métrages confondus), ce qui permettrait d'évaluer à 44.117 le nombre de participants à cette édition de la manifestation. La représentativité des 2.539 questionnaires recueillis serait alors de 5,8\%. 
8. Les pays les plus représentés au sein de cette enquête sont le Brésil (25,2 \%), le Mexique $(19,4$ $\%)$, l'Argentine $(1,9 \%)$, la Colombie $(8,1 \%)$ et la Fédération de Russie $(5,0 \%)$. Les résultats relatifs à la participation par nationalité sont assez concordants avec les statistiques émises par Unifrance à propos de la nationalité des internautes qui ont téléchargé des films sur la plateforme de My French Film Festival, à l'exception de la Chine, sous-représentée dans l'enquête (seulement 7 questionnaires ont été remplis dans ce pays, alors qu'on l'a vu, c'est dans ce pays que le nombre de visionnages est le plus important).

9. La plupart des festivals cherchent en effet à diversifier leurs lieux de projection à l'intérieur de la ville où ils sont implantée, afin d'aller à la rencontre de nouveaux publics. On peut proposer l'exemple du Festival International du Film de la Rochelle, initialement implanté à la Coursive, et qui peu à peu multiplie les lieux de projection à travers la ville (dans les salles de cinéma commerciales, à l'Espace culturel municipal, à la Médiathèque, à La Sirène/Espace musiques actuelles...). Les mêmes enjeux de démocratisation culturelle sont poursuivis lorsque cette délocalisation se joue à l'extérieur de la commune. Le festival Ciné Jeunes, essentiellement localisé à Saint-Quentin, dans l'Oise, délocalise ainsi ses projections dans 30 sites à travers le département.

10. La décentralisation au niveau national et international concerne essentiellement les plus grandes manifestations cinématographiques. Ainsi, le Festival International du film de Cannes organise-t-il une décentralisation de sa programmation à Paris et Marseille, mais aussi à Genève, Bruxelles et Rome.

\section{RÉSUMÉS}

On voit fleurir depuis quelques années des festivals de cinéma en ligne, manifestation proposant, par le biais d'un site internet, le téléchargement des œuvres cinématographiques programmées dans le cadre dudit événement. Si ce développement - inattendu - du secteur festivalier sur le terrain des nouvelles technologies ouvre des horizons inespérés en faveur d'une circulation accélérée des œuvres cinématographiques, le cyber-festivalier devient, dans cette nouvelle configuration, un individu, seul devant sa machine, alors même que les festivals se sont historiquement positionnés en faveur d'une réception collective, conviviale, des films. L'objet de cet article est d'interroger cette évolution, d'évaluer la capacité de ce nouveau type de manifestation à demeurer un élément structurant face à une communauté devenue virtuelle donc fluctuante. Comment repenser la fonction de médiateur telle que l'ont historiquement pensé les festivals dans ce contexte de circulation accélérée et démultipliée des œuvres que propose leur avènement sur la Toile?

For the last few years, online film festivals have flourished. These events offer, through a website, the download of programmed films. This - unexpected - development of the world of festivals in the field of new technologies gives a tremendous boost to the circulation of cinematographic works; but at the same time, the "cyber festival fan" involves one individual, alone in front of his machine, even though, historically speaking, festivals have clearly adopted positions in favour of a collective, convivial, reception of works. The purpose of this article is to question this change, to evaluate online festivals' ability to remain a structuring element for an online community, virtual, therefore fluctuating. How should we rethink the festivals' role of mediator in this 
context of accelerated and exacerbated circulation of works resulting from their advent on the Web?

INDEX

Keywords : festival, online festival, circulation, Internet

Mots-clés : festival, festival en ligne, circulation, Internet

\section{AUTEURS}

\section{CHRISTEL TAILLIBERT}

Université Nice Sophia Antipolis

Maître de conférences

taillibe@unice.fr 